\title{
Kinetic feedback computation for polynomial systems to achieve weak reversibility and minimal deficiency
}

\author{
György Lipták ${ }^{2}$, Gábor Szederkényi ${ }^{1,2}$ and Katalin M. Hangos ${ }^{2,3}$ \\ ${ }^{1}$ Faculty of Information Technology, Péter Pázmány Catholic University, Práter u. 50/a, H-1083 Budapest, Hungary \\ ${ }^{2}$ Process Control Research Group, MTA SZTAKI, Kende u. 13-17, H-1111 Budapest, Hungary \\ ${ }^{3}$ Department of Electrical Engineering and Information Systems, University of Pannonia, Egyetem u. 10, \\ H-8200 Veszprém, Hungary \\ Email: szederkenyi@itk.ppke.hu, lipgyorgy@gmail.com, hangos@scl.sztaki.hu
}

\begin{abstract}
In this paper, an optimization based state feedback design is proposed for polynomial models that transforms an open-loop system into weakly reversible kinetic form with minimal deficiency, if possible. Therefore, the suggested method is able to decide whether the deficiency zero property and weak reversibility of the closed loop system (that guarantees a robust stability property) is achievable by the given feedback structure. The approach integrates feedback design and previous computational methods for computing dynamically equivalent realizations of kinetic systems. The method assumes a linear input structure of the open loop system, and uses a polynomial feedback constructed from the monomials of the original system possibly extended by new ones. The proposed method is illustrated on two simple examples.

Index Terms - Computational methods; Optimization; Biomolecular systems
\end{abstract}

\section{INTRODUCTION}

The field of feedback controller design for nonlinear systems has been continuously developing in recent decades, because of its practical importance and challenging theoretical nature. It is well-known that the utilization of the physical and/or structural specialties of different nonlinear system classes greatly helps in obtaining theoretically well-grounded, powerful and practically still feasible control methods: e.g. we have sound methods of nonlinear feedback design for smooth input-affine systems [1], flat systems [2], Hamiltonian or port-Hamiltonian systems [3], [4], or that for Euler-Lagrange systems [5].

Deterministic kinetic systems with mass action kinetics or simply chemical reaction networks (CRNs) form a wide class of nonnegative polynomial systems. CRNs are able to produce all the important qualitative phenomena present in nonlinear systems, so they form a rich-enough sub-class there. A recent survey shows [6] that CRNs are also widely used in other areas than chemical reaction kinetics or process systems that include biological applications, such as to model the dynamics of intracellular processes and metabolic or cell signalling pathways [7].

The theory of chemical reaction networks has significant results relating network structure and the qualitative properties of the corresponding dynamics [8], [9]. However, the network structure corresponding to a given dynamics is generally not unique [10]. Recently, optimization-based computational methods were proposed for dynamically equivalent network structures with given preferred properties (see, e.g. [11]-[14]).

Therefore, the general purpose of our work is to construct polynomial feedback controllers to polynomial systems to achieve a kinetic closed loop system with given advantageous structural properties. In [15], the problem of obtaining a kinetic closed loop system was addressed in the framework of mixed integer linear programming. In this contribution, the deficiency of the preferably weakly reversible closed loop system is also minimized that is practically more interesting and, at the same time, a more complex computational task.

\section{UNDERLYING NOTIONS AND METHODS}

Polynomial systems form a wide and well-studied class of smooth nonlinear systems that have important applications in diverse engineering fields, such as (bio)chemical engineering, process systems engineering, transportation engineering, etc. Within these fields, positive (or nonnegative) polynomial systems are often considered that is dictated by the physical meaning (e.g. pressure, concentration or the vehicle number/density) of the signals.

The notion of positive systems builds upon the essential nonnegativity of a function $f=\left[\begin{array}{lll}f_{1} & \ldots & f_{n}\end{array}\right]^{T}:[0, \infty)^{n} \rightarrow$ $\mathbb{R}^{n}$, that holds if, for all $i=1, \ldots, n, f_{i}(x) \geq 0$ for all $x \in[0, \infty)^{n}$, whenever $x_{i}=0$ [16].

An autonomous nonlinear system defined on the nonnegative orthant $[0, \infty)^{n}=\overline{\mathbb{R}}_{+}^{n} \subset \mathcal{X}$

$$
\dot{x}=f(x), x(0)=x_{0}
$$

where $f: \mathcal{X} \rightarrow \mathbb{R}^{n}$ is locally Lipschitz, $\mathcal{X}$ is an open subset of $\mathbb{R}^{n}$ and $x_{0} \in \mathcal{X}$ is nonnegative (or positive) when the nonnegative (or positive) orthant is invariant for the dynamics (1). This property holds if and only if $f$ is essentially nonnegative.

\section{A. Kinetic systems, their dynamics and structure}

Deterministic kinetic systems with mass action kinetics or simply chemical reaction networks (CRNs) form a wide class of nonnegative polynomial systems, that are able 
to produce all the important qualitative phenomena (e.g. stable/unstable equilibria, oscillations, limit cycles, multiplicity of equilibrium points and even chaotic behavior) present in the dynamics of nonlinear processes [6]. The structure of CRNs is well characterized by a weighted directed graph, called the reaction graph, and by their complex composition matrix.

The problem of kinetic realizability of polynomial vector fields was first examined and solved in [17] where it was shown, that the necessary and sufficient condition for kinetic realizability of a polynomial vector field is that all coordinates functions of $f$ in (1) must have the form

$$
f_{i}(x)=-x_{i} g_{i}(x)+h_{i}(x), i=1, \ldots, n
$$

where $g_{i}$ and $h_{i}$ are polynomials with nonnegative coefficients. It's easy to prove that kinetic systems are nonnegative.

The ODE form: If the condition (2) is fulfilled for a polynomial dynamical system, then it can always be written into the form

$$
\dot{x}=Y \cdot A_{k} \cdot \psi(x),
$$

where $x \in \mathbb{R}^{n}$ is the vector of state variables, $Y \in \mathbb{Z}_{\geq 0}^{n \times m}$ with distinct columns is the so-called complex composition matrix, $A_{k} \in \mathbb{R}^{m \times m}$ contains the information corresponding to the weighted directed graph, the reaction graph, of the reaction network (see below). As it will be visible later, the generally non-unique factorization (3) is particularly useful for prescribing structural constraints using optimization. According to the original chemical meaning of this system class, the state variables represent the concentrations of the chemical species denoted by $X_{i}$, i.e. $x_{i}=\left[X_{i}\right]$ for $i=1, \ldots, n$. Moreover, $\psi: \mathbb{R}^{n} \mapsto \mathbb{R}^{m}$ is a mapping given by

$$
\psi_{j}(x)=\prod_{i=1}^{n} x_{i}^{Y_{i j}}, \quad j=1, \ldots, m .
$$

$A_{k}$ is a column conservation matrix (i.e. the sum of the elements in each column is zero) defined as

$$
\left[A_{k}\right]_{i j}=\left\{\begin{array}{cl}
-\sum_{l=1, l \neq i}^{m} k_{i l}, & \text { if } i=j \\
k_{j i}, & \text { if } i \neq j .
\end{array}\right.
$$

Note that $A_{k}$ is also called as the Kirchhoff matrix of the network.

The complexes are formally defined as nonnegative linear combinations of the species in the following way:

$$
C_{i}=\sum_{j=1}^{n} Y_{j i} X_{j}, i=1, \ldots, n
$$

Note, that a column (let's say column $i$ ) of the matrix $Y$ may be equal to the zero vector. In such a case, node $C_{i}$ is called the zero complex.
The reaction graph: The weighted directed graph (or reaction graph) of kinetic systems is $G=(V, E)$, where $V=\left\{C_{1}, C_{2}, \ldots, C_{m}\right\}$ and $E$ denote the set of vertices and directed edges, respectively. The directed edge $\left(C_{i}, C_{j}\right)$ (also denoted by $C_{i} \rightarrow C_{j}$ ) belongs to the reaction graph if and only if $\left[A_{k}\right]_{j, i}>0$. In this case, the weight assigned to the directed edge $C_{i} \rightarrow C_{j}$ is $\left[A_{k}\right]_{j, i}$.

The dynamic properties of a CRN depend on some of the structural properties of the reaction graph. A CRN is called weakly reversible if whenever there exists a directed path from $C_{i}$ to $C_{j}$ in its reaction graph, then there exists a directed path from $C_{j}$ to $C_{i}$. In graph theoretic terms, this means that all components of the reaction graph are strongly connected components.

Deficiency and the zero deficiency theorem: The deficiency [9] is a fundamental property of a CRN. Its notion depends on the notion of a reaction vector corresponding to $C_{i} \rightarrow C_{j}$, and denoted by $e_{k}$ :

$$
e_{k}=[Y]_{\cdot, j}-[Y]_{\cdot, i}, k=1, \ldots, r,
$$

where $[Y]_{,, i}$ denotes the $i$ th column of $Y$ and $r$ is the number of reactions. The rank of a reaction network denoted by $s$ is the rank of the set of vectors $H=\left\{e_{1}, e_{2} \ldots, e_{r}\right\}$. The stoichiometric subspace, denoted by $S$, is defined as $S=\operatorname{span}\left\{e_{1}, \ldots, e_{r}\right\}$.

The deficiency $d$ of a reaction network is defined as [9]: $d=m_{n i}-l-s$, where $m_{n i}$ is the number of nonisolated (i.e. reacting) vertices in the reaction graph, $l$ is the number of linkage classes (graph components) and $s$ is the rank of the reaction network. The deficiency is a very useful measure for studying the dynamical properties of reaction networks and for establishing parameterindependent global stability conditions.

The Deficiency Zero Theorem [9] shows a very robust stability property of a certain class of kinetic systems. It says that deficiency zero weakly reversible networks possess well-characterizable equilibrium points, and independently of the weights of the reaction graph (i.e. as long as the positive elements of the $A_{k}$ matrix remain positive) they are at least locally stable with a known logarithmic Lyapunov function that is also independent of the system parameters. According to the so-called Global Attractor Conjecture (to which no counterexample has been found), weakly reversible deficiency zero CRNs are globally stable (within the positive orthant). This conjecture has been proved for CRNs containing one linkage class [18]. Moreover, weakly reversible deficiency zero models are input-to-state stable with respect to the off-diagonal elements of $A_{k}$ as inputs [19], it is straightforward to asymptotically stabilize them by additional feedback [20], and it is possible to construct efficient state observers for them [21].

\section{B. Dynamical equivalence of $C R N s$}

It is a known result of chemical reaction network theory that a reaction graph corresponding to a given set of 
kinetic ODEs is generally not unique. We will use the degree of freedom given by this phenomenon for feedback design. Using the notation $M=Y \cdot A_{k}$, equation (3) can be written in the form

$$
\dot{x}=M \cdot \psi(x),
$$

where $M$ contains the coefficients of the monomials in the polynomial ODE (3) describing the time-evolution of the state variables. We call two reaction networks given by the matrix pairs $\left(Y^{(1)}, A_{k}^{(1)}\right)$ and $\left(Y^{(2)}, A_{k}^{(2)}\right)$ dynamically equivalent, if

$$
Y^{(1)} A_{k}^{(1)} \psi^{(1)}(x)=Y^{(2)} A_{k}^{(2)} \psi^{(2)}(x)=f(x), \quad \forall x \in \overline{\mathbb{R}}_{+}^{n}
$$

where for $i=1,2, Y^{(i)} \in \mathbb{R}^{n \times m_{i}}$ have nonnegative integer entries, $A_{k}^{(i)}$ are valid Kirchhoff matrices, and

$$
\psi_{j}^{(i)}(x)=\prod_{k=1}^{n} x_{k}^{\left[Y^{(i)}\right]_{k j}}, \quad i=1,2, j=1, \ldots, m_{i} .
$$

In this case, $\left(Y^{(i)} A_{k}^{(i)}\right)$ for $i=1,2$ are called dynamically equivalent realizations of the corresponding kinetic vector field $f$. It is also appropriate to call $\left(Y^{(1)}, A_{k}^{(1)}\right)$ a (dynamically equivalent) realization of $\left(Y^{(2)}, A_{k}^{(2)}\right)$ and vice versa.

\section{Computing weakly reversible realizations with minimal} deficiency

In this subsection, the results of [14] are briefly summarized that will be used for feedback design in a straightforward way. The basis of the method is the recognition that for weakly reversible networks, it is enough to maximize the number of linkage classes (i.e. graph components) to minimize deficiency. An additional applied known result is that a reaction graph is weakly reversible if and only if there is a strictly positive vector in the kernel of the Kirchhoff matrix $A_{k}$. Then, the goal of the optimization task is to allocate complexes between the possible maximal number of linkage classes while maintaining dynamical equivalence.

The constraints for dynamical equivalence are easy to write as follows:

$$
\begin{aligned}
\tilde{Y} \cdot \tilde{A}_{k} & =\tilde{M} \\
\sum_{i=1}^{m}\left[\tilde{A}_{k}\right]_{i j} & =0, j=1, \ldots, m \\
0 \leq\left[\tilde{A}_{k}\right]_{i j} & \leq 1 / \epsilon, i, j=1, \ldots, m, i \neq j
\end{aligned}
$$

where $\tilde{Y}$ and $\tilde{M}$ are the known complex composition matrix and coefficient matrix of the right hand side of the polynomial differential equations, respectively. The off-diagonal elements of the Kirchhoff matrix $\tilde{A}_{k}$ are unknowns, and $\epsilon$ is a sufficiently small number used for bounding the elements of $A_{k}$. This bounding is technically needed because the final optimization problem will contain integer variables as well. It can be easily shown that the maximal possible number of linkage classes in any computed realization is $m-s$ [14]. To track the graph nodes among the graph components (linkage classes), binary variables $\gamma_{i k}$, for $i=1, \ldots m, k=1, \ldots m-s$ are introduced: $\gamma_{i k}=1$ if and only if $C_{i}$ belongs to the $k$-th linkage class. We also introduce other auxiliary variables $\theta_{k} \in[0,1]$, for $k=1, \ldots, m-s$, where $\theta_{k}=0$ indicates that the $k$-th linkage class is empty. The complete partitioning of the complexes between linkage classes is expressed by the constraints:

$$
\begin{aligned}
& \sum_{k=1}^{m-s} \gamma_{i k}=1, \quad i=1, \ldots, m \\
& \sum_{i=1}^{m} \gamma_{i k}-\epsilon \theta_{k} \geq 0, \quad k=1, \ldots, m-s \\
& -\sum_{k=1}^{m} \gamma_{i k}+\frac{1}{\epsilon} \theta_{k} \geq 0, \quad k=1, \ldots, m-s \\
& \gamma_{i k} \in\{0,1\}, \quad i=1, \ldots, m, k=1, \ldots, m-s \\
& \theta_{k} \in[0,1], \quad k=1, \ldots, m-s .
\end{aligned}
$$

To ensure weak reversibility, we use an $m \times m$ Kirchhoff matrix $\Phi$ that is a column-scaled version of $A_{k}$, i.e. $\Phi=$ $\tilde{A}_{k} \cdot \operatorname{diag}(b)$, where $b \in \mathbb{R}^{m}$ is a strictly positive vector in the kernel of $\tilde{A}_{k}$. It is clear that the positions of zero and non-zero elements in $\tilde{A}_{k}$ and $\Phi$ are the same, and therefore reaction graph encoded by $\tilde{A}_{k}$ is weakly reversible if and only if the $m$-dimensional vector containing only ones, i.e. $\left[\begin{array}{llll}1 & 1 & \ldots & 1\end{array}\right]^{T} \in \mathbb{R}^{m}$ belongs to the kernel of $\Phi$. Let us add the following constraint set to the problem:

$$
\begin{aligned}
& \sum_{\substack{l=1 \\
l \neq i}}^{m} \Phi_{i l}=\sum_{\substack{l=1 \\
l \neq i}}^{m} \Phi_{l i} \\
& \Phi_{i j} \leq \frac{1}{\epsilon}\left(\gamma_{i k}-\gamma_{j k}+1\right) \\
& \Phi_{i j} \geq \epsilon\left[\tilde{A}_{k}\right]_{i j} \\
& \Phi_{i j} \leq \frac{1}{\epsilon}\left[\tilde{A}_{k}\right]_{i j} \\
& i, j=1, \ldots, m, i \neq j, \quad k=1, \ldots, m-s .
\end{aligned}
$$

The constraints in (13) ensure the following key properties: 1) identical structure of $\Phi$ and $A_{k}, 2$ ) weak reversibility of the reaction graph corresponding to $\Phi$ and $\left.A_{k}, 3\right)$ there cannot be directed edges between different linkage classes. Finally, the uniqueness of solution can be enforced by the following constraint:

$$
\begin{aligned}
& \sum_{j=1}^{i-1} \gamma_{j k} \geq \sum_{l=k+1}^{m-s} \gamma_{i l}, \\
& i=1, \ldots, m, k=1, \ldots, m-s, k \leq i .
\end{aligned}
$$

By minimizing the following objective function, the deficiency is also minimized (through maximizing the number of linkage classes):

$$
V(\theta)=\sum_{k=1}^{m-s} \theta_{k}
$$


It is visible that constraints (11)-(14) together with the objective function in (15) form a standard mixed integer linear programming (MILP) problem.

\section{Feedback computation}

In this section, the optimization problems for the design of static and dynamic kinetic feedback are described. First, the autonomous system model (8) will be extended with a simple linear input structure.

\section{A. Open loop model form}

We assume that the equations of the open loop polynomial system with linear input structure are given as

$$
\dot{x}=M \cdot \psi_{1}(x)+B u,
$$

where $x \in \mathbb{R}^{n}$, is the state vector, $u \in \mathbb{R}^{p}$ is the input, $\psi_{1} \in \mathbb{R}^{n} \rightarrow \mathbb{R}^{m_{1}}$ contains the monomials of the open-loop system, $B \in \mathbb{R}^{n \times p}$ and $M \in \mathbb{R}^{n \times m_{1}}$.

The problem that we will study is to design a static or dynamic monomial feedback such that the closed loop system is kinetic, and there exists a realization that fulfills a required property (in this particular case, weak reversibility with minimal deficiency).

\section{B. Static feedback design}

We assume a polynomial feedback of the form

$$
u=K \cdot \bar{\psi}(x),
$$

where $\bar{\psi}(x)=\left[\psi_{1}^{T}(x) \psi_{2}^{T}(x)\right]^{T}$ with $\psi_{2} \in \mathbb{R}^{n} \rightarrow \mathbb{R}^{m_{2}}$ containing possible additional monomials for the feedback, $B \in \mathbb{R}^{n \times p}$, and $K \in \mathbb{R}^{p \times\left(m_{1}+m_{2}\right)}$. The closed-loop system can be written as

$$
\dot{x}=M \cdot \psi_{1}(x)+B K\left[\begin{array}{l}
\psi_{1}(x) \\
\psi_{2}(x)
\end{array}\right] .
$$

We can partition $K$ into two blocks as $K=\left[K_{1} K_{2}\right]$, where $K_{1} \in \mathbb{R}^{p \times m_{1}}$ and $K_{2} \in \mathbb{R}^{p \times m_{2}}$. Using this notation, the closed loop dynamics is given by

$$
\dot{x}=\underbrace{\left[\begin{array}{ll}
M+B K_{1} & B K_{2}
\end{array}\right]}_{\bar{M}}\left[\begin{array}{l}
\psi_{1}(x) \\
\psi_{2}(x)
\end{array}\right]=\bar{M} \cdot \bar{\psi}(x) .
$$

The aim is to set the closed loop coefficient matrix $\bar{M}$ such that it defines a kinetic system with $\bar{\psi}$. It is clear from subsection II-A that this is possible if and only if $\bar{M}$ can be factorized as $\bar{M}=\bar{Y} \cdot \bar{A}_{k}$ where $\bar{Y} \in \mathbb{Z}_{\geq 0}^{n \times\left(m_{1}+m_{2}\right)}$, and $\bar{A}_{k} \in \mathbb{R}^{\left(m_{1}+m_{2}\right) \times\left(m_{1}+m_{2}\right)}$ is a valid Kirchhoff matrix.

Based on constructing the so-called canonical realization of a kinetic system [17], we can give a simple method to generate matrix $\bar{Y}$ (and thus $\psi_{2}$ given by such monomials that do not appear in (16)) using the monomials of the open loop system as as described in [15]. After constructing $\bar{Y}$, the kinetic property, minimal deficiency and weak reversibility of the controlled system can be achieved if the MILP problem defined by (11)-(14) and (15) can be solved for $\tilde{A}_{k}=\bar{A}_{k}$ substituting $\tilde{Y}=\bar{Y}$ and $\tilde{M}=\bar{M}$.
Thus, the feedback gain computation and the search for weakly reversible realizations with minimal deficiency of the closed loop system has been integrated into one MILP optimization problem. It has to be noted that while $\tilde{M}$ is assumed to be known in (11), $\bar{M}$ contains unknowns, namely the feedback parameters $K_{1}$ and $K_{2}$, but this does not change the linear nature of the constraints and the MILP computation framework is still applicable.

\section{Computation of dynamic feedbacks}

To increase the degrees of freedom in transforming a polynomial system to kinetic form via feedback, it is a straightforward idea to apply a dynamic extension. In this case, let us write the equations of the open-loop system as

$$
\dot{x}^{(1)}=M_{11} \psi_{1}\left(x^{(1)}\right)+B u,
$$

where $x^{(1)} \in \mathbb{R}^{n}, M_{11 \in \mathbb{R}^{n \times m_{1}}}, \psi_{1}: \mathbb{R}^{n} \rightarrow \mathbb{R}^{m_{1}}, B \in \mathbb{R}^{n \times p}$, and $u \in \mathbb{R}^{p}$. Let us give the equations of the dynamic extension as

$$
\dot{x}^{(2)}=M_{21} \psi_{1}\left(x^{(1)}\right)+M_{22} \psi_{2}(x),
$$

where $x^{(2)} \in \mathbb{R}^{k}, M_{21} \in \mathbb{R}^{k \times m_{1}}, M_{22} \in \mathbb{R}^{k \times m_{2}}$. Moreover,

$$
x=\left[\begin{array}{l}
x^{(1)} \\
x^{(2)}
\end{array}\right] \in \mathbb{R}^{n+k}, \bar{\psi}(x)=\left[\begin{array}{c}
\psi_{1}\left(x^{(1)}\right) \\
\psi_{2}(x)
\end{array}\right],
$$

where $\psi_{2}: \mathbb{R}^{n+k} \rightarrow \mathbb{R}^{m_{2}}$. Let us again use a monomial feedback in the form $u=K \bar{\psi}(x)=K_{1} \psi_{1}+K_{2} \psi_{2}$, where $K_{1} \in \mathbb{R}^{p \times m_{1}}, K_{2} \in \mathbb{R}^{p \times m_{2}}$, and $K=\left[\begin{array}{ll}K_{1} & K_{2}\end{array}\right]$. The equations of the closed loop system are given by

$$
\dot{x}=\left[\begin{array}{ll}
M_{11}+B K_{1} & B K_{2} \\
M_{21} & M_{22}
\end{array}\right] \cdot \bar{\psi}(x)=\bar{M} \cdot \bar{\psi}(x)
$$

The feedback gain computation, the weak reversibility and minimal deficiency constraint is completely analogous to the static feedback case described in subsection III-B with the only exception that we have more unknowns (i.e. decision variables) in matrices $M_{21}$ and $M_{22}$ giving generally more degrees of freedom to solve the feedback design problem.

\section{EXAMPLES}

In the following, we demonstrate our feedback design algorithms with two simple examples.

\section{A. Designing a dynamic feedback structure for a polyno- mial system}

Let us consider the following polynomial system

$$
\begin{aligned}
& \dot{x}_{1}=-x_{1} x_{2}+2 x_{2}^{2} x_{3} \\
& \dot{x}_{2}=x_{1} x_{2}-4 x_{2}^{2} x_{3}-x_{2} x_{3}^{2}+u_{1} \\
& \dot{x}_{3}=6+x_{1} x_{2}-3 x_{2}^{2} x_{3}+u_{2}
\end{aligned}
$$

It is easy to see from (26) that for $u_{1}=0, u_{2}=0$, the system has no equilibrium points in the nonnegative 
orthant. Using the notations of section III, we have:

$$
\begin{aligned}
& \psi_{1}\left(x^{(1)}\right)=\left[\begin{array}{llll}
1 & x_{1} x_{2} & x_{2}^{2} x_{3} & x_{2} x_{3}^{2}
\end{array}\right]^{T}, \\
& M_{11}=\left[\begin{array}{rrrr}
0 & -1 & 2 & 0 \\
0 & 1 & -4 & -1 \\
6 & 1 & -3 & 0
\end{array}\right], \quad B=\left[\begin{array}{ll}
0 & 0 \\
1 & 0 \\
0 & 1
\end{array}\right]
\end{aligned}
$$

For a dynamical feedback, let us introduce one new variable $x^{(2)}=x_{4}$, and an additional monomial as follows: $\psi_{2}(x)=\left[x_{3}^{2} x_{4}\right]$. Then, after performing the procedure presented in subsection III-C, we find that the MILP optimization problem is feasible, and

$$
\begin{aligned}
& K=\left[\begin{array}{rrrrr}
1 & 0 & 2 & 0 & 0 \\
2 & 0 & 1 & 0 & -10
\end{array}\right], M_{21}=\left[\begin{array}{llll}
3 & 0 & 0 & 1
\end{array}\right] \\
& M_{22}=[-5]
\end{aligned}
$$

This means that the feedback: $u_{1}=2 x_{2}^{2} x_{3}, u_{2}=x_{2}^{2} x_{3}-$ $10 x_{3}^{2} x_{4}$, and the dynamic extension: $\dot{x}_{4}=3+x_{2} x_{3}^{2}-5 x_{3}^{2} x_{4}$ results in a closed loop system that has a weakly reversible realization with zero deficiency. Therefore, the controlled system has bounded trajectories in the positive orthant and moreover, it is globally stable with a known logarithmic Lyapunov function. The resulting weakly reversible reaction graph of the closed loop system is depicted in Fig. 1.

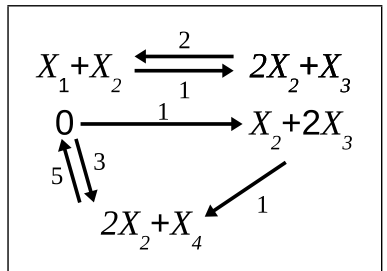

Figure 1. Weakly reversible kinetic structure of the closed loop system

B. Designing a static feedback structure for the Lorenz system

Let us consider the extended version of the well-known 3 -dimensional Lorenz system by linear input terms as an open loop polynomial system

$$
\begin{aligned}
& \dot{x}=\sigma(y-x)+u_{1} \\
& \dot{y}=x(\rho-z)-y+u_{2} \\
& \dot{z}=x y-\beta z+u_{3}
\end{aligned}
$$

Let the parameter values be $\sigma=10, \rho=28, \beta=8 / 3$ that are known to lead to chaotic behavior for $u=0$, that is also clearly visible from Fig. 3. It is important to note that the above model is not kinetic.
Using the notations of section III, we have:

$$
\begin{aligned}
& \psi_{1}(x, y, z)=\left[\begin{array}{lllll}
x & y & z & x z & x y
\end{array}\right]^{T}, \\
& M_{11}=\left[\begin{array}{rrrrr}
-10 & 10 & 0 & 0 & 0 \\
28 & -1 & 0 & -1 & 0 \\
0 & 0 & -2.6667 & 0 & 1
\end{array}\right] \text {, } \\
& B=\left[\begin{array}{lll}
1 & 0 & 0 \\
0 & 1 & 0 \\
0 & 0 & 1
\end{array}\right]
\end{aligned}
$$

In designing the feedback we are going to use the original monomials only and we are not using dynamical extension. Then, after solving the MILP problem described in subsection III-B, we find that the problem is feasible, and

$$
K=\left[\begin{array}{rrrrr}
9.9 & -9.8 & 0.1 & -0.1 & -0.1 \\
-27.9 & 0.8 & 0.1 & 1.1 & -0.1 \\
0 & 0.2 & 2.5667 & -0.2 & -0.9
\end{array}\right]
$$

The obtained feedback structure results in a closed loop system that has a weakly reversible realization with zero deficiency. The resulting weakly reversible reaction graph of the closed loop system is depicted in Fig. 2, while Fig. 4 illustrates the stable behavior of the controlled system. Note that the above feedback completely changes

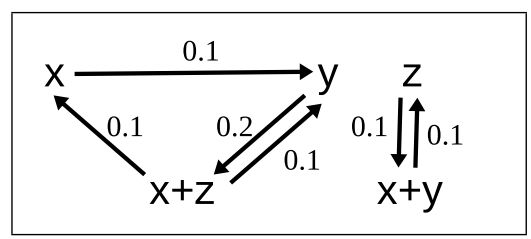

Figure 2. Weakly reversible kinetic structure of the closed loop system

the coefficients of the nonlinear terms in the model by leaving its monomial terms unchanged.

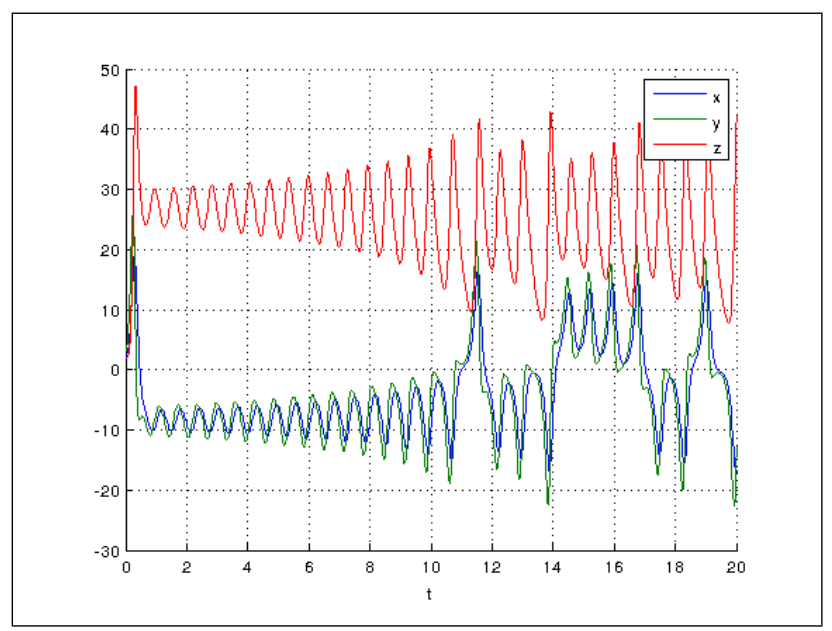

Figure 3. Chaotic behavior of the open-loop Lorenz system 


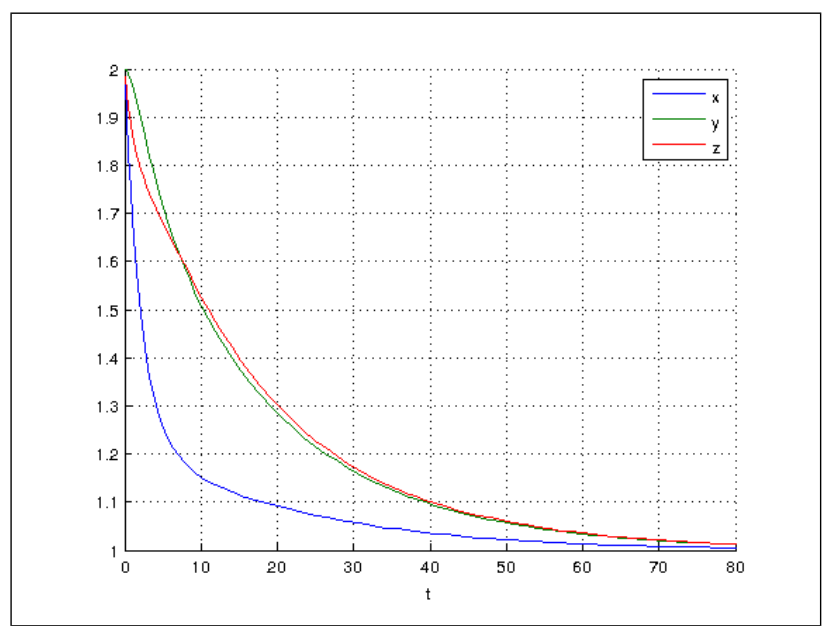

Figure 4. Time-domain behaviour of the controlled Lorenz system

\section{Conclusions}

A novel optimization based state feedback design method was proposed in this paper for polynomial systems that transforms the closed loops system into kinetic form with minimal deficiency and weak reversibility. Weak reversibility ensures the boundedness of the trajectories in the positive orthant, while global stability can be achieved in the zero deficiency case. Both static and dynamic feedback designs are considered. The computational method uses MILP for jointly determining the feedback parameters and the preferred dynamically equivalent realization of the closed loop system as a kinetic system.

The controller structure assumes a linear input structure of the open loop system, and uses a polynomial feedback constructed from the monomials of the original system possibly extended by new ones.

The proposed method is illustrated by two simple examples, including a Lorenz system with chaotic behavior in the open-loop case.

\section{ACKNOWLEDGEMENTS}

This research has been supported by the Hungarian National Research Fund through grants NF104706 and K83440.

\section{REFERENCES}

[1] A. Isidori, Nonlinear Control Systems. Springer, Berlin, 1999.

[2] J. Levine, Analysis and Control of Nonlinear Systems: A Flatness-Based Approach. Springer, 2009.

[3] J. Clemente-Gallardo and J. Scherpen, "Relating Lagrangian and Hamiltonian framework for LC circuits," IEEE Transactions on Circuits and Systems, vol. 50, pp. 1359-1363, 2003.

[4] A. van der Schaft, L2-Gain and Passivity Techniques in Nonlinear Control. Berlin: Springer, 2000.

[5] R. Ortega, A. Loría, P. J. Nicklasson, and H. Síra-Ramírez, Passivity-Based Control of Euler-Lagrange Systems: Mechanical, Electrical and Electromechanical Applications. SpringerVerlag, 1998.

[6] D. Angeli, "A tutorial on chemical network dynamics," European Journal of Control, vol. 15, pp. 398-406, 2009
[7] J. Haag, A. Wouver, and P. Bogaerts, "Dynamic modeling of complex biological systems: a link between metabolic and macroscopic description," Mathematical Biosciences, vol. 193, pp. $25-49,2005$

[8] F. Horn and R. Jackson, "General mass action kinetics," Archive for Rational Mechanics and Analysis, vol. 47, pp. 81-116, 1972.

[9] M. Feinberg, "Chemical reaction network structure and the stability of complex isothermal reactors - I. The deficiency zero and deficiency one theorems," Chemical Engineering Science, vol. 42 (10), pp. 2229-2268, 1987.

[10] G. Craciun and C. Pantea, "Identifiability of chemical reaction networks," Journal of Mathematical Chemistry, vol. 44, pp. 244$259,2008$.

[11] G. Szederkényi, "Computing sparse and dense realizations of reaction kinetic systems," Journal of Mathematical Chemistry, vol. 47, pp. 551-568, 2010.

[12] G. Szederkényi, K. M. Hangos, and Z. Tuza, "Finding weakly reversible realizations of chemical reaction networks using optimization," MATCH Communications in Mathematical and in Computer Chemistry, vol. 67, pp. 193-212, 2012, iF: 3.29 (2010).

[13] G. Szederkényi, J. R. Banga, and A. A. Alonso, "Inference of complex biological networks: distinguishability issues and optimization-based solutions," BMC Systems Biology, vol. 5, p. $177,2011$.

[14] M. D. Johnston, D. Siegel, and G. Szederkényi, "Computing weakly reversible linearly conjugate chemical reaction networks with minimal deficiency," Mathematical Biosciences, vol. 241, pp. 88-98, 2013.

[15] G. Szederkényi, G. Lipták, J. Rudan, and K. M. Hangos, "Optimization-based design of kinetic feedbacks for nonnegative polynomial systems," in Proceedings of the 9th IEEE Conferene on Computational Cybernetics, 2013, pp. 67-72.

[16] V. Chellaboina, S. P. Bhat, W. M. Haddad, and D. S. Bernstein, "Modeling and analysis of mass-action kinetics - nonnegativity, realizability, reducibility, and semistability," IEEE Control Systems Magazine, vol. 29, pp. 60-78, 2009.

[17] V. Hárs and J. Tóth, "On the inverse problem of reaction kinetics," in Qualitative Theory of Differential Equations, ser. Coll. Math. Soc. J. Bolyai, M. Farkas and L. Hatvani, Eds. North-Holland, Amsterdam, 1981, vol. 30, pp. 363-379.

[18] D. F. Anderson, "A proof of the Global Attractor Conjecture in the single linkage class case," SIAM Journal on Applied Mathematics, vol. accepted, p. to appear, 2011, http://arxiv.org/abs/1101.0761.

[19] M. Chaves, "Input-to-state stability of rate-controlled biochemical networks," SIAM Journal on Control and Optimization, vol. 44, pp. 704-727, 2005.

[20] E. Sontag, "Structure and stability of certain chemical networks and applications to the kinetic proofreading model of T-cell receptor signal transduction," IEEE Transactions on Automatic Control, vol. 46, pp. 1028-1047, 2001.

[21] M. Chaves and E. D. Sontag, "State-estimators for chemical reaction networks of Feinberg-Horn-Jackson zero deficiency type," European Journal of Control, vol. 8, pp. 343-359, 2002. 